\title{
Interference Minimization in 5G Physical-Layer Network Slicing
}

\author{
Marco Zambianco and Giacomo Verticale
}

\begin{abstract}
The interference resulting from densification of access points and the coexistence of different numerologies within the same spectrum severely hinders inter-slice isolation. We propose a slice allocation policy that enforces inter-slice isolation by minimizing the inter-slice interference suffered by each virtual operator. In detail, we design a binary quadratic non-convex optimization problem that minimizes i) the interslice interference generated by interfering base stations and ii) the inter-slice interference generated by the multiplexing of spectrum slices having different numerologies. We also provide a heuristic algorithm to render the solution scalable in practical scenarios. We assess the performance of both approaches by evaluating the signal-to-interference-plus-noise ratio (SINR) associated to each slice through simulations. Results reveal that the heuristic algorithm provides a solution comparable with the optimal one on different minimization scenarios. Moreover, a considerable SINR improvement is observed with respect to a base-line scheme that does not account for inter-slice interference.
\end{abstract}

Index Terms-Radio spectrum management, resource management, interference

\section{INTRODUCTION}

RAN slicing makes it possible to "slice" the physical network resources, managed by a network owner (NO), among multiple Mobile Virtual Network Operators (MVNOs) enabling the deployment of different services on top of the same network infrastructure. A network slice is commonly defined as a set of network functions and the resources to run these network functions, forming a complete instantiated logical network to meet the specific network characteristics required by the application [1]. In other words, each network slice can be considered as an independent network entity tailored to guarantee a set of service level agreement (SLA) requirements.

RAN slicing entails additional effort in the design of efficient and effective slicing policies for two main reasons: i) radio spectrum is a scarce resource and ii) slice performance is related to the portion of spectrum to which it has access. In fact, when different slices are multiplexed within the same bandwidth, each slice performance is affected by the number of radio resources that are simultaneously shared with other slices. Slice isolation becomes an essential requirement since it ensures that coexisting slices do not have any mutual impact on each other's performance. From a radio perspective, spectrum slices must not interfere with one another so that every MVNO can schedule its own users without affecting the performance of users in other slices.

Due to the densification of the access points and to the employment of beamforming, inter-cell interference (ICI) across base stations (BS) is going to be a common issue that needs to be taken into account in the design of efficient RAN slicing policies. In addition, a traditional single-numerology access scheme consisting in a homogeneous division of the time-frequency resources is no longer suitable to embrace the different service requirements characterizing $5 \mathrm{G}$ applications. Instead, a mixed-numerology scheme is proposed to increase the flexibility and reliability in the accommodation of services. Specifically, differently from the conventional orthogonal frequency division multiplexing (OFDM), this scheme supports the allocation of heterogeneous frequency-time resources, denoted as resource blocks (RBs), composed by different subcarrier spacing and transmission intervals within the same transmission frame. On one hand, such technique provides a common underlying physical to multiplex various services, on the other hand, it affects the inter-slice isolation due to the inter-numerology interference (INI) generated between adjacent non-orthogonal RBs [2]. The main contributions of this paper are summarized as follows:

- We provide a mathematical model to represent a mixednumerology RB grid as composed of single-numerology RBs.

- We design a slicing allocation policy that enforces interslice isolation between different MVNOs. More precisely, we formulate a non-convex binary quadratic optimization problem that minimizes the inter-slice interference generated when i) multiple MVNOs are simultaneously allocated to the same spectrum slice on interfering BSs and ii) spectrum slices composed of RBs of different numerologies are simultaneously multiplexed.

- We propose a heuristic algorithm that outputs an RB allocation policy requiring a lower amount of time than the optimal solution. The scheme provides a solution that balances the trade-off between the two interference sources and achieves a performance level that is comparable to the optimal one.

- We assess the benefit deriving from inter-slice interference minimization by computing the average SINR associated to each slice throughout simulations. In detail, we show a significant SINR improvement provided by both approaches with respect to a base-line allocation policy that does not enforce inter-slice isolation.

The rest of the paper is organized as follows. In Section II, we describe the considered system model. Section III discusses the formulation of the optimization problem that minimizes inter-slice interference. In section IV, we show the simulation results. Finally, the conclusions are drawn in Section V.

\section{RELATED WORK}

RAN slicing has received a growing interest in the last few years from the research community. An overview of the 
key concept of this topic as well as the related challenges is provided by several surveys such as [3][4][5]. Most work in this field proposes RAN slicing schemes that leverage the design of an orchestration framework [6], an admission control policy [7], and a resource management policy [8]. The goal of the aforementioned work is to ensure that RAN slices are characterized by an efficient sharing of the resources and a suitable customization to accommodate each service. However, the proposed slicing schemes are agnostic of the underlying physical layer, since they do not show how to quantitatively allocate radio resources to each slice. In addition, slice isolation is not directly considered in the system model but it is empirically evaluated by simulations.

Following these observations, recent work has proposed slicing schemes to address these issues. In [9], the authors propose a resource sharing agreement policy that accounts for the correlation among data to reduce the number of dropped packets. The same authors design an interference management module to enable resource isolation. However, they consider fractional frequency reuse, thus limiting the slice flexibility and lowering the system efficiency. In [10], the authors design an inter-MVNO RB sharing policy that allocates resources based on the interference level affecting various users. However, since multiple RBs can be shared among multiple MVNO, advanced communication techniques like CoMP cannot be applied. In [11], the authors propose a slicing scheme that allocates RBs capable of satisfying the SLA requirements. Such policy selects RBs from overprovisioned slices and assigns them to the under-performing slices. The authors of [12] propose an online approach based on genetic algorithm to slice resources, that does not require any pre-knowledge about the utility model. In [13], a RB allocation scheme is designed such that the number of satisfied slices in term of SLA requirements is maximized with a minimum number of allocated RBs. However, the approaches proposed in [11] [12] [13] ensure slice isolation only for a single BS case, whereas multi-cell scenario remains uncovered. The authors of [14] describe how to enforce slice isolation by minimizing inter-slice interference between MVNOs deployed on interfering BSs. However, the solution does not account for the multi-numerology case. The authors of [15] propose an auction-based policy that allocates RBs to different slices according to the users bids, where each slice is modelled with a different numerology type. However, although a mixednumerology scenario is considered, their auction-based scheme neglects the effect of inter-numerology interference.

Differently from these papers, we design an allocation policy of the radio resources that enforces inter-slice isolation among RAN slices employing different numerologies. More precisely, the proposed scheme accounts for two interference sources that affect the isolation among MVNOs: i) interslice interference derived from interfering cells and ii) internumerology interference derived from the simultaneous multiplexing of RBs having heterogeneous subcarrier spacing.

\section{SYSTEM MODEL}

In this section we introduce the considered RAN architecture and we describe the models employed to describe a mixed-numerology resource grid as well as inter-slice interference.

\section{A. RAN slicing model}

We consider a $5 \mathrm{G}$ RAN of bandwidth $L$ consisting of $B$ macro BSs equipped with Remote Radio Heads (RRHs), and a centralized pool of Base Band Units (BBUs). The various RRHs are shared among a set of $M$ MVNOs that provide different network services. Each MVNO is a logical standalone radio interface that schedules the available radio resources among its users in order to fulfill the SLA requirements. We consider a mixed-numerology OFDMA scheme such that each spectrum slice assigned to MVNO $m$ on the BS $b$, denoted as $\ell_{m, b}$, can be modelled as a set of RBs, each one representing a fixed number of contiguous subcarriers, allocated over $T$ time slots. The duration of each time slot has a granularity tailored to the radio environment statistics (e.g. shorter time slots are suited for urban environments, whereas longer ones could be employed to model RANs in rural scenarios) and it is considered as constant in our problem. The NO manages the whole RAN infrastructure and periodically assigns spectrum slices $\ell_{m, b}$ among the MVNOs in each BS following the slice assignment policy $S_{m, b}=\left\{\ell_{m^{\prime}, b} \cap \ell_{m, b}=\emptyset \wedge \sum_{m \in M} \ell_{m, b}=\right.$ $\left.L, \forall m, m^{\prime} \neq m \in M, \forall b \in B\right\}$. In this context, the RAN slicing architecture can be considered as composed by a twolevel scheduler. The higher level is represented by the MVNOs schedulers, whereas the lower level is represented by the NO scheduler. The latter allocates spectrum slices, according to $S_{m, b}$, to each MVNO in order to provide better scheduling opportunities for their users.

Every MVNO $m$ is modelled with a different numerology type $\mu_{m}$ suited to satisfy the application requirements. Consequently, the associated spectrum slices are composed by RBs having subcarrier spacing $\Delta f_{m}=2^{\mu_{m}} \cdot 15 \mathrm{kHz}$. Concerning the RB allocation over time, as imposed by 3GPP specification [16], we consider a synchronized transmission in each time subframe of length $1 \mathrm{~ms}$ to prevent symbol misalignment. In Table I we report the available numerologies as well as the $\mathrm{RB}$ structure in 5G NR standard. According to such RB characterization, the resulting resource grid $R$ is composed by $N_{R B}$ RBs of different subcarrier spacing that are multiplexed over $T$ time slots based on $S_{m, b}$.

TABLE I

Numerology Structure in 5G NeW Radio Standard

\begin{tabular}{cccc}
\hline$\mu$ & $\Delta f(\mathrm{kHz})$ & Subcarriers per RB & RB per subframe \\
\hline 0 & 15 & 12 & 1 \\
1 & 30 & 12 & 2 \\
2 & 60 & 12 & 4 \\
3 & 120 & 12 & 8 \\
4 & 240 & 12 & 16 \\
\hline
\end{tabular}

\section{B. Mixed-numerology resource grid model}

Differently from single numerology systems, the total number of RBs in mixed-numerology schemes that can be allocated varies according to the bandwidth occupied by each RB. In 
detail, the higher is the bandwidth per $\mathrm{RB}$, the lower is the total number of resources that can be allocated in a particular transmission frame. Therefore, a homogeneous resource grid cannot provide an effective way to meet this behavior, since the bandwidth spanned by each RB depends on the specific numerology used in that time slot. To address this issue, we rely on the observation that each numerology is an integer multiple of a minimum subcarrier spacing, thus each RB can be seen as a set of contiguous RBs with minimum numerology. Specifically, let $\mu_{\min }=\operatorname{argmin}\left\{\Delta f_{m}\right\}$ be the smallest numerology in the system. The numerology associated to each MVNO $m \in M$ can be expressed as $F_{m}=2^{\left(\mu_{m}-\mu_{m i n}\right)}$ contiguous RBs of numerology $\mu_{\min }$. Note that this property holds only if the number of subcarriers in each RB is constant, since it requires the $\mathrm{RB}$ bandwidth to be directly proportional to the subcarrier spacing.

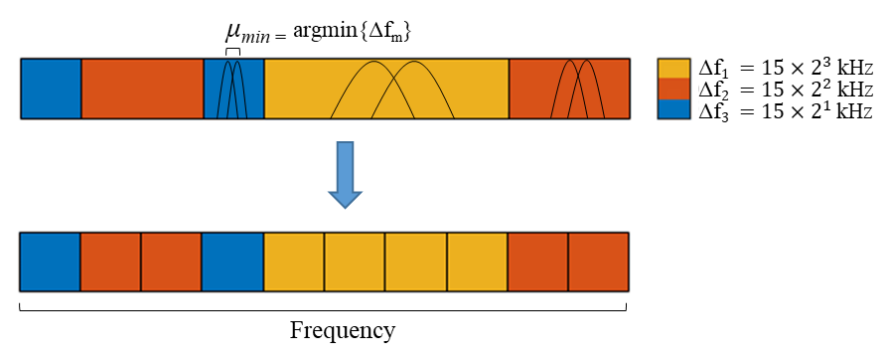

Fig. 1. Mixed-numerology RB grid representation.

Leveraging this alternative numerology representation, depicted in Fig. 1, we define the resource grid $\tilde{R}$ as the set of $\tilde{N}_{R B}$ RBs with constant numerology $\mu_{\min }$ over $T$ time slots. This allows us to model the RB assignment by means of the allocation indicator function $x_{m, b, n, t} \in\{0,1\}$. In detail, the latter is defined for any MVNO $m \in M$ with numerology $\mu_{m}$ on $\mathrm{BS} b \in B$ as:

1) $\mathrm{RB}(n, t) \in \tilde{R}$ is allocated if

$$
x_{m, b, n, t}=1
$$

and, if $F_{m}>1$,

$$
x_{m, b, n+i, t}=0, \quad 1 \leq i \leq\left(F_{m}-1\right)
$$

2) $\mathrm{RB}(n, t) \in \tilde{R}$ is not allocated if

$$
x_{m, b, n, t}=0
$$

and, if $F_{m}>1$,

$$
x_{m, b, n+i, t}=0, \quad 1 \leq i \leq\left(F_{m}-1\right) .
$$

In other words, the RB allocation indicator represents each numerology as a group of $F_{m}$ contiguous RBs of numerology $\mu_{\text {min }}$ by flagging the position of the first RB and zeroing the subsequent ones. We do not need to specify additional conditions for the RB allocation over time domain as we assume that they are aggregated to provide a synchronized transmission every time slot $t$. Given this model, the allocation policy can be designed similarly to a single-numerology access scheme. The advantage of this representation is to formulate more easily the objective function (see Section IV).

\section{Inter-numerology interference model}

Inter-numerology interference is caused by the loss of the orthogonality between subcarriers within the same OFDM symbol. In fact, OFDM samples at both the transmitter and the receiver side are corrupted by the spectral emissions of subcarriers having different bandwidth. To analytically model this behavior, we rely on the formulation proposed in [2] to compute the interference between subbands of different numerologies. We consider two subbands $B_{1}$ and $B_{2}$ of numerology type $\mu_{1}$ and $\mu_{2}$ with $\Delta f_{1}<\Delta f_{2}$, each one composed by $Z_{1}$ and $Z_{2}$ subcarriers. For each subband, we denote the Discrete Fourier transform (DFT) symbol and cyclic prefix (CP) length as $N^{\left(B_{i}\right)}$ and $N_{C P}^{\left(B_{i}\right)}$, respectively. The INI power suffered from $k$ th subcarrier of subband $B_{1}$ due to $v$ th interfering subcarrier of subband $B_{2}$ at spectral distance $\Delta k=k-v$ can be approximated as

$$
\begin{aligned}
P_{v}^{\left(B_{1}\right)}(k) \approx & \frac{\left|H^{\left(B_{2}\right)}(v)\right|^{2}}{N^{\left(B_{2}\right)} N^{\left(B_{1}\right)}}\left[\left|\frac{\sin \left[\frac{\pi}{N^{\left(B_{1}\right)}} \Delta k^{\left(B_{1}\right)} \gamma N_{T}^{\left(B_{2}\right)}\right]}{\sin \left(\frac{\pi}{N^{\left(B_{1}\right)}} \Delta k^{\left(B_{1}\right)}\right)}\right|^{2}\right. \\
& \left.+\gamma\left|\frac{\sin \left[\frac{\pi}{N^{\left(B_{1}\right)}} \Delta k^{\left(B_{1}\right)} N_{T}^{\left(B_{2}\right)}\right]}{\sin \left[\frac{\pi}{N^{\left(B_{1}\right)}} \Delta k^{\left(B_{1}\right)}\right]}\right|^{2}\right],
\end{aligned}
$$

where $\gamma=\left\lfloor N^{\left(B_{1}\right)} / N_{T}^{\left(B_{2}\right)}\right\rfloor, N_{T}^{\left(B_{2}\right)}=N^{\left(B_{2}\right)}+N_{C P}^{\left(B_{2}\right)}$ and $H^{\left(B_{2}\right)}(v)$ is the frequency response of the $v$ th subcarrier of subband $B_{2}$. Similarly, the INI power caused by the $v$ th subcarrier of subband $B_{1}$ on the $k$ th subcarrier of subband $B_{2}$ can be approximated as

$$
P_{v}^{\left(B_{2}\right)}(k) \approx \frac{\left|H^{\left(B_{1}\right)}(v)\right|^{2}}{N^{\left(B_{2}\right)} N^{\left(B_{1}\right)}}\left|\frac{\sin \left[\frac{\pi}{N^{\left(B_{1}\right)}} \Delta k^{\left(B_{2}\right)} N^{\left(B_{2}\right)}\right]}{\sin \left[\frac{\pi}{N^{\left(B_{1}\right)}} \Delta k^{\left(B_{2}\right)}\right]}\right|^{2},
$$

where $H^{\left(B_{1}\right)}(v)$ is the frequency response of the $v$ th subcarrier of subband $B_{1}$. From the above formulations, it is possible to observe the asymmetric behavior of the interference power that is mutually generated by each subband. Specifically, the wider is the spectral gap between different numerologies (in other words, $\Delta f_{1}<<\Delta f_{2}$ ), the higher is the interference generated on the subband with the smaller numerology type.

\section{Beamforming interference model}

We model the radio coverage area with a hexagonal-cell network layout following the guidelines for $5 \mathrm{G}$ dense macrocell scenarios as dictated by ITU [17]. In detail, each BS provides radio coverage on a $120^{\circ}$ sector that is modelled with a hexagonal area. The various BSs are grouped as triplets in order to provide an aggregated coverage of $360^{\circ}$ and are located on different sites that are at distance $d$ one from the other. Every site orientation is such that sectors on different sites do not directly face each other on the same coverage direction. A representation of the considered RAN topology is shown in Fig. 2. According to such network layout, we do not consider any intra-site interference. Differently, we account for the interference between BSs that are located on neighbor sites at distance $d$ and have substantially overlapping coverage. This results in sectors that can interfere with at most 4 other BSs. Following this assumption, we define the 


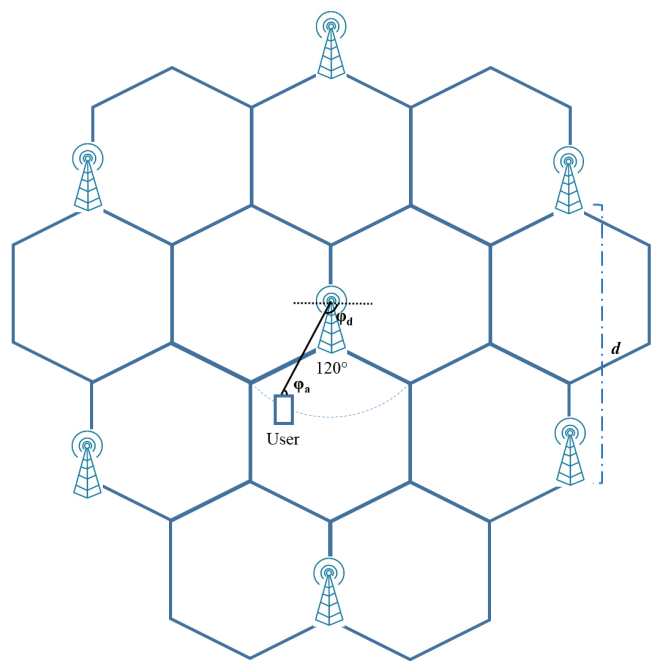

Fig. 2. RAN topology model.

adjacency matrix $\mathbf{Y}$ such that $y_{b, b^{\prime}}=1$ if BSs $b$ and $b^{\prime}$ interfere each other and $y_{b, b^{\prime}}=0$ otherwise. Each sector serves the users within its coverage area by means of beamforming transmission and spatially multiplexes $U$ beams towards their locations. Beamforming impacts on the isolation of network slices for two reasons: i) the transmission of highly directional signals enables the deployment of closer BSs that boost the network capacity. However, cell-edge users could suffer from severe interference generated by beams, on interfering BSs, directed on the boundaries of their coverage area ii) the benefit of interference reduction in the regions closer to each $\mathrm{BS}$ is limited by the effect of inter-numerology interference as its interference power is comparable to the inter-cell one.

We measure the inter-slice interference experienced by each user as the number of RBs that are simultaneously assigned to multiple MVNOs on interfering cells. In detail, when the RBs allocated to every MVNO span the same spectrum slice during time slot $t$ on interfering BSs, inter-slice interference is absent. Conversely, if RBs are assigned to more than one MVNO in the same time slot $t$, inter-slice isolation cannot be ensured and users scheduled in each slice generate interference towards users belonging to other MVNOs. In other words, if a spectrum slice is accessed only by a single MVNO, users on different slices are not affected by inter-slice interference, otherwise the level of interference generated depends on the number of overlapped RBs assigned to different MVNO in each time slot.

We consider a statistical approach to model the inter-slice interference power generated by the various beams that are transmitted by each sector. Specifically, as proposed in other works [18][19][20], we assume that the main lobe of each beam of beamwidth $\theta_{t} \in[0,2 \pi / 3]$ provides a constant transmission gain $g_{t}=Q$ and it is directed towards the intended user, whereas side lobes are approximated with an overall transmission gain of $g_{t}=q$ with $q<Q$ and are pointed in other directions. Similarly, we model each user with steerable directional antennas that have constant receiving gain $g_{r}=Q$ for beamwidth $\theta_{r} \in[0,2 \pi]$ and $g_{r}=q$ otherwise. Assuming perfect beam alignment between serving sector and user, the total transmission gain of the serving link can be computed as $G_{s}=g_{t} \cdot g_{r}=Q \cdot Q$. Similarly, we model the interference gain $G_{i}$ on the serving link due to the transmission on interfering sectors, as a discrete random variable. Specifically, according to the angle of departure (AoD) $\varphi_{d}$ of the various beams from the interfering sectors and the angle of arrivals (AoA) $\varphi_{a}$ of such beams on the antenna pattern of a generic victim user, the total transmission gain can assume one of the values in the alphabet $X=\{Q Q, Q q, q q\}$. In this regard, we consider both AoD and AoA to be independent and uniformly distributed within the interval $0 \leq \varphi_{d} \leq 2 \pi / 3$ and $0 \leq \varphi_{a} \leq 2 \pi$ respectively, thus the probability density function $p_{G_{i}}(X)$ can be computed as

$$
\begin{aligned}
p_{G_{i}}(Q Q) & =\frac{\theta_{t}}{2 \pi / 3} \cdot \frac{\theta_{r}}{2 \pi} \\
p_{G_{i}}(Q q) & =\frac{2 \pi / 3-\theta_{t}}{2 \pi / 3} \cdot \frac{\theta_{r}}{2 \pi}+\frac{\theta_{t}}{2 \pi / 3} \cdot \frac{2 \pi-\theta_{r}}{2 \pi} \\
p_{G_{i}}(q q) & =\frac{2 \pi / 3-\theta_{t}}{2 \pi / 3} \cdot \frac{2 \pi-\theta_{r}}{2 \pi} .
\end{aligned}
$$

\section{E. Signal-to-interference model}

We describe the large-scale propagation fading, $G_{p l}$, by means of Close-In (CI) path loss model, that is relevant for a wide range of frequencies as well as $5 \mathrm{G}$ propagation scenarios [21]. The inter-slice interference power is composed by two terms: i) ICI generated by beams on adjacent sectors and ii) INI generated by the multiplexing of spectrum slices having different numerologies. Therefore, let $m \in M$ be a MVNO whose spectrum slice is composed by $Z_{m}$ subcarriers, the signal-to-interference-plus-noise ratio (SINR) associated to a generic user located at distance $r$ from the serving BS $s$, with transmission power $P_{s}$, and at distance $r^{\prime}$ from a neighbor interfering $\mathrm{BS} i$, with transmission power $P_{i}$, can be computed as

$$
S I N R=\frac{P_{s} G_{s} G_{p l}(r)}{P_{i n i} G_{p l}(r)+P_{i c i} G_{p l}\left(r^{\prime}\right)+W_{m}}
$$

where

$$
\begin{aligned}
& P_{i n i}=G_{s} \sum_{k=1}^{Z_{m}}\left(\sum_{\substack{m^{\prime} \in M \\
m \neq m^{\prime}}} \sum_{v=1}^{Z_{m^{\prime}}} P_{v}^{B_{m}}(k)\right) \text {, } \\
& P_{i c i}=P_{i} \sum_{u=1}^{U} G_{i}^{u}
\end{aligned}
$$

and $W_{m}$ is noise power over the bandwidth composed by $Z_{m}$ subcarriers. To better clarify the above formulations, we remark that the INI power in a subband due to multiple adjacent subbands of different numerologies is computed following two steps. First, we compute the interference power caused by adjacent subbands over the subcarriers of the considered subband using (1) and (2). Then, we compute the total interference power by summing the interference contribution affecting each subcarrier. Concerning ICI power, we recall that $G_{i}$ is generated according to the probability distribution (3). 


\section{INTER-SLICE INTERFERENCE ANALYSIS}

In this section we discuss the proposed optimization problem that minimizes inter-slice interference. Specifically, we compute the optimal RB allocation by accounting for both interference terms in the objective function. Moreover, we propose a heuristic approach to overcome the computational complexity of the optimal formulation.

\section{A. Problem formulation}

Among all the feasible slice assignment policies $S_{b, m}$, we focus on the design of a slice allocation policy that enforces inter-slice isolation. We achieve this goal by minimizing the interference affecting each MVNO. Specifically, we formulate an optimization problem that computes an $\mathrm{RB}$ allocation capable of minimizing inter-slice interference generated by ICI and INI.

To achieve the first objective, we recall that inter-MVNO interference is absent when the same spectrum slice is simultaneously assigned to the same MVNO on interfering BSs. Formally, a RB $(n, t)$ of numerology type $\mu_{m}$ on the resource grid $\tilde{R}$ is uniquely allocated to MVNO $m$ on two interfering $\mathrm{BS} b$ and $b^{\prime}$ if and only if

$$
x_{m, b, n, t}=x_{m, b^{\prime}, n, t}=1
$$

and, if $F_{m}>1$,

$$
x_{m, b, n+i, t}=x_{m, b^{\prime}, n+i, t}=0 \quad 1 \leq i \leq\left(F_{m}-1\right) .
$$

From this definition, it easy to understand that RBs allocated in this way allow the corresponding MVNO to access a specific spectrum slice in the same time slot from different BSs, thus enabling a coherent and coordinated transmission.

To address the INI between different slices, we rely on the model described in Section III to design an allocation policy that reduces the INI power on each slice. Employing INI power equations (1) and (2) results in a complicated, non-linear objective function that cannot be easily solved. Therefore, we propose a simpler objective function based on the RB allocation indicator function previously defined. From (1) and (2), we observe that INI power mainly depends on two factors: i) the spectral distance $\Delta k$ between the victim subcarrier and the interfering subcarrier and ii) the relative difference between the numerology types of the subcarriers in the two subbands. Specifically, the wider is their difference (that is reflected by their DFT size $N^{\left(B_{1}\right)}$ and $N^{\left(B_{2}\right)}$ ) the higher is the interference generated over the subcarriers with the smaller numerology type. Consequently, RBs of similar numerologies produce less interference when they are allocated close to each other than RBs with very different numerologies. This result is also supported by authors in [22], where they show the signal-to-interference ratio (SIR) degradation as function of the numerology gap between different subbands.

Following this observation, our policy is designed to minimize the number of contiguously allocated RBs of different numerologies over the same transmission interval. To formalize this concept, first we provide the definition of contiguously allocated RBs based on the RB allocation indicator function.
An RB $(n, t)$ of numerology type $\mu_{m}$ is contiguously allocated next to an $\mathrm{RB}\left(n^{\prime}, t\right)$ of numerology type $\mu_{m^{\prime}}$ if and only if

$$
x_{m, b, n, t}=x_{m^{\prime}, b, n-F_{m^{\prime}, t}}=1
$$

and, if $F_{m^{\prime}}>1$,

$$
x_{m, b^{\prime}, n-F_{m^{\prime}}+i, t}=0 \quad 1 \leq i \leq\left(F_{m^{\prime}}-1\right) .
$$

Based on this, given an RB $(n, t)$ of numerology type $\mu_{m}$, the INI power generated by a contiguous $\mathrm{RB}\left(n^{\prime}, t\right)$ of numerology type $\mu_{m^{\prime}}$, is minimized if and only if

$$
\left|\mu_{m}-\mu_{m^{\prime}}\right| \leq\left|\mu_{i}-\mu_{j}\right| \quad \forall i, j \in M .
$$

Intuitively, the designed policy aims at allocating RBs with the same numerology over a contiguous spectrum slice based on the assignment policy $S_{b, m}$. In this scenario, the INI power derives only from the interference generated between RBs at the boundaries of each spectrum slice.

To quantitatively model this behavior, we design a penalty function $f_{p}\left(m, m^{\prime}\right)$ that, for every MVNOs pair $\left\{m, m^{\prime}\right\}$, associates a cost $c_{m, m^{\prime}}$ to the contiguous allocation of the related RBs. In detail, $f_{p}\left(m, m^{\prime}\right)$ expresses how much the numerologies of each MVNOs pair differ in terms of subcarrier spacing. Consequently, the higher is the numerology gap $k_{m, m^{\prime}}=\left|\mu_{m}-\mu_{m^{\prime}}\right|$ between each pair, the higher is the $\operatorname{cost} c_{m, m^{\prime}}$. Formally, we define the penalty function as

$$
f_{p}\left(m, m^{\prime}\right)=\left\{\begin{array}{lll}
0 & \text { if } & k_{m, m^{\prime}}=0 \\
1 & \text { if } & k_{m, m^{\prime}}=1 \\
C \cdot k_{m, m^{\prime}} & \text { if } & k_{m, m^{\prime}}>1
\end{array}\right.
$$

where the constant $C \geq 1$ allows to tune the penalty associated to the contiguous allocation of RBs that are very different in terms of subcarrier spacing. We note that (7) does not penalize the contiguous allocation of RBs with equal numerologies since they do not interfere each other. Instead, it assigns a low penalty when RBs of similar numerologies (i.e., $k_{m, m^{\prime}}=1$ ) are contiguously allocated, whereas it assigns a higher penalty, depending on $C$, in the other cases. Based on the structure of (7), we define the penalty matrix $\mathbf{W}$ such that each element $w_{m, m^{\prime}}$ is equal to $c_{m, m^{\prime}}$.

We remark that the proposed design is sub-optimal with respect to the direct minimization of (1) and (2), since it is not able to model the complete INI dynamic. In fact, our approach does not account neither for the INI asymmetry nor for the INI generated by other RBs that are not directly located next to the victim RB. Nevertheless, we assessed by means of numerical analysis that our simplified model provides a good approximation of the INI behavior. Specifically, the analysis showed that the gap, between the solution obtained with the problem formulation discussed in Section III and the best solution extracted by an exhaustive search of all feasible RB allocations, can be easily neglected.

\section{B. Optimal solution}

Leveraging the ideas of uniquely allocated RBs and contiguously allocated RBs, we design a binary quadratic optimization problem that minimizes inter-slice interference due to ICI 
and INI. To balance the contribution of the two interference sources, we introduce a weight coefficient, denoted as $\alpha$, that allows to prioritize the minimization of one term over the other. Formally, we have:

$$
\begin{aligned}
& \min _{x}(1-\alpha) \cdot \\
& \left(-\sum_{m \in M} \sum_{t=1}^{T} \sum_{n=1}^{\tilde{N}_{R B}} \sum_{\substack{b \in B \\
b^{\prime} \neq b \\
b^{\prime} \in B}} y_{b, b^{\prime}}\left(F_{m} x_{m, b, n, t} x_{m, b^{\prime}, n, t}\right)\right)+ \\
& \alpha\left(\sum_{b \in B} \sum_{m \in M} \sum_{m^{\prime} \in M} \sum_{n=1}^{\tilde{N}_{R B}} \sum_{t=1}^{T} w_{m, m^{\prime}} x_{m, b, n, t} x_{m^{\prime}, b, n-F_{m^{\prime}, t}}\right)
\end{aligned}
$$

subject to

$$
\begin{aligned}
& \sum_{t=1}^{T} \sum_{n=1}^{\tilde{N}_{R B}} x_{m, b, n, t}=\frac{\ell_{m, b}}{F_{m}}, \forall b \in B, \forall m \in M \\
& \sum_{i=0}^{F_{m}-1} x_{m, b, n-i, t} \leq 1, \forall(n, t) \in \tilde{R}, \forall b \in B, \forall m \in M \\
& \sum_{m \in M} \sum_{i=0}^{F_{m}-1} x_{m, b, n-i, t} \leq 1, \forall(n, t) \in \tilde{R}, \forall b \in B \\
& x_{m, b, n, t} \in\{0,1\}, \forall(n, t) \in \tilde{R}, \forall b \in B, \forall m \in M .
\end{aligned}
$$

In equation (8), the objective function includes two terms, whose minimization priority is dictated by the parameter $\alpha \in[0,1]$. In particular, when $\alpha=0$, the proposed formulation targets ICI minimization only. Conversely, when $\alpha=1$, only INI is minimized. For all other values of $\alpha$, the resulting $\mathrm{RB}$ allocation provides an interference minimization that is a weighted trade-off between the two objectives. Specifically, we minimize the inter-slice interference due to ICI by minimizing the number of non-uniquely allocated RBs to multiple MVNOs on interfering BSs. Note that $F_{m}$ is needed to equalize the contribution of each uniquely allocated RB according to its numerology. In fact, following the proposed model of mixednumerology RB grid, a RB with numerology $\mu_{m}$ accounts as $F_{m}$ RBs of numerology $\mu_{\min }$, thus it provides a reward that is $F_{m}$ times higher when it is uniquely allocated (in the particular case of all RBs with equal numerology, $F_{m}$ can be neglected since it assumes a constant value). Differently, we minimize the inter-slice interference due to INI by minimizing the number of contiguous RBs having different numerologies on every BS. Intuitively, the effect of this simultaneous minimization is that, for every uniquely allocated RB to MVNO $m$, the objective function pays a penalty $w_{m, m^{\prime}}$ based on the numerology gap from the RB of MVNO $m^{\prime}$ that is contiguously allocated.

Problem constraints define the set of feasible allocation policy solutions according to the employed mixed-numerology RB model. In detail, constraint (9) ensures that all MNVOs receive a number of RBs expressed as groups of $F_{m}$ contiguous RBs according to the spectrum slice $\ell_{m, b}$. Constraints (10) and (11) account for the multiplexing of RBs with different numerologies represented through the $\mathrm{RB}$ allocation indicator.
More preciselyy, constraint (10) characterizes the numerology $\mu_{m}$ of each MVNO $m$ by imposing groups of $F_{m}$ contiguous RBs with numerology $\mu_{m i n}$. Constraint (11) guarantees that each group of $F_{m}$ contiguous RBs is allocated to one MVNO only. Finally, (12) ensures that the RB allocation indicator can only assume binary values.

\section{Solution complexity and scalability}

The complexity of problem (8) is NP-hard. In fact, as the matrix associated to the quadratic form of the ICI term is indefinite (the proof is provided by authors in [14] that proposed a similar problem formulation), it is known that the related problem complexity is NP-hard [23]. This is sufficient to prove that also (8) is NP-hard as the solutions instances of the problem in [14] are a subset of the solutions of problem (8) obtained when every MVNO has the same numerology.

Regarding the scalability of the problem with respect to the number of BSs and MVNOs, we note that ICI term scales quadratically with number of interfering links between the available BSs. The adjacency matrix $\mathbf{Y}$ defines the BSs couples that require the minimization of the number of nonuniquely assigned RBs. When each BS interferes each other, the couples cardinality grows with square of the number of BSs, since every interfering link produces the couples $y_{b, b^{\prime}}$ and $y_{b^{\prime}, b}$. This observation has an important implication from a practical perspective as the addition of more BSs does not necessarily degrade the computational time performance to an unacceptable level. In fact, the scalability can be close to linear if $\mathbf{Y}$ is sparse, which corresponds to a realistic scenario where each BS interferes only with a subset of the total number of BSs belonging the same RAN.

A similar reasoning can be applied to the scalability with respect to the number of MVNOs when the penalty matrix $\mathbf{W}$ is considered. The computational complexity scales quadratically with number of MVNOs as they produces more RBs couples of different numerologies. More precisely, such quadratic relationship is actually related to the number of new pairs $w_{m, m^{\prime}}$ and $w_{m^{\prime}, m}$ that are generated by multiplexing more MVNOs of different numerologies. However, in practical systems, the number of numerology types is limited, hence the computational complexity increases with a behavior close to linear once all the available numerologies are employed.

\section{Heuristic solution}

The time complexity required for the optimal $\mathrm{RB}$ allocation policy computed with (8) denies a online deployment on systems that require frequents updates of the assignment policy $S_{m, b}$. For this reason, we propose a heuristic algorithm to speed up the convergence time of the original problem at the cost of a sub-optimal solution. As (8) generates different solutions based on $\alpha$, it is not feasible to provide a heuristic for each value. Therefore, we design a scheme to approximate the set of optimal solutions providing a balanced trade-off between the number uniquely allocated RBs and the number of contiguously allocated RBs of different numerologies. Intuitively, this corresponds to the range of $\alpha$ values that are sufficiently low to minimize the number of non-uniquely assigned RBs 
and, at the same, sufficiently high to minimize the number of contiguously assigned RBs of different numerologies.

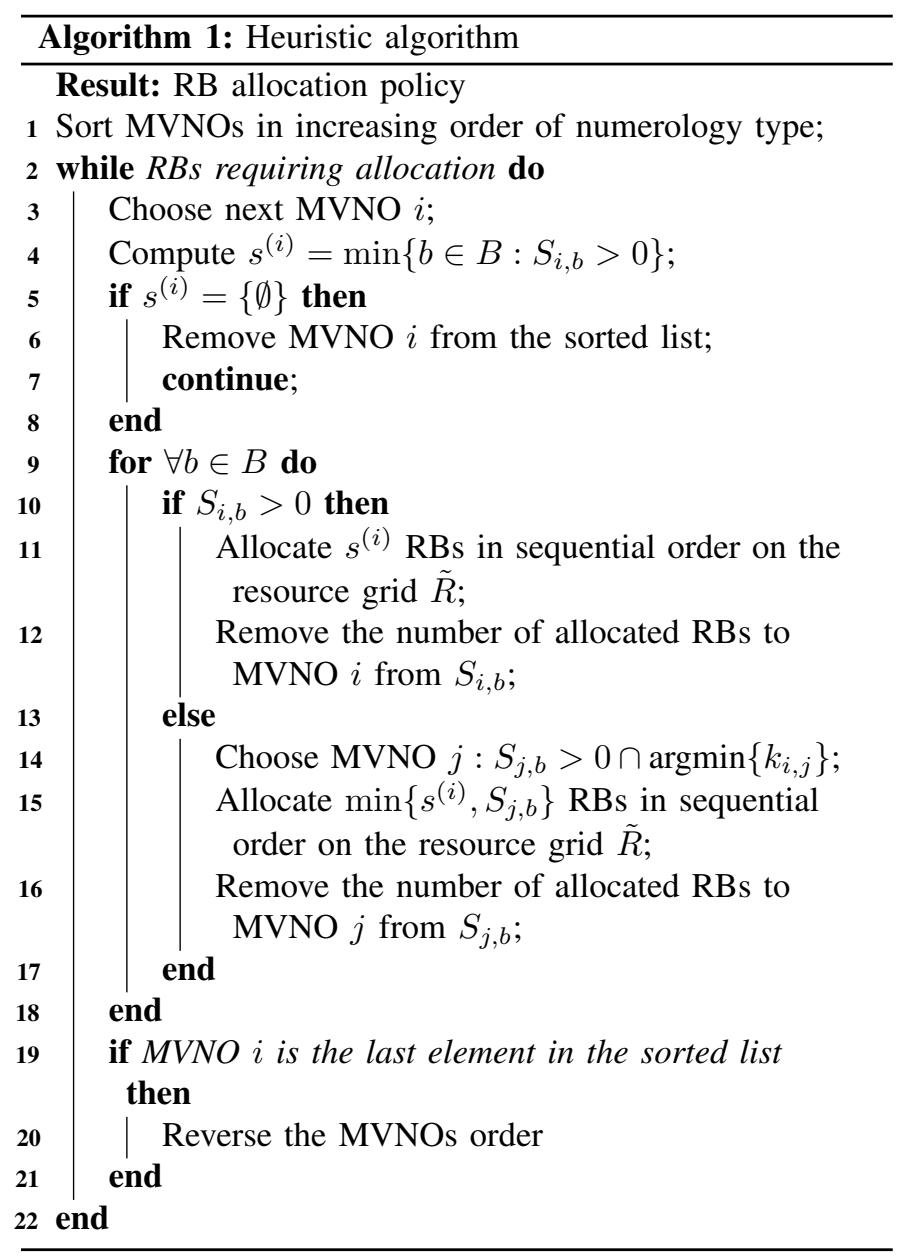

Following this assumption, we present the pseudo-code of the proposed heuristic scheme in Alg. 1. The key idea behind the algorithm is to allocate clusters of $\mathrm{RBs}$, belonging to same MVNO, that are aligned on similar frequency-time slots in every BS. This has the benefit of INI mitigation as RBs with the same numerology are more likely to be contiguously allocated and, at the same, of ICI mitigation as such RBs simultaneously share similar spectrum regions (i.e. they are aligned) on different BSs. In detail, RBs are allocated starting from the MVNOs with smaller numerologies as they are more affected by INI (line 1). The allocation scheme is repeated until every $\mathrm{RB}$ is allocated (line 2). Starting from the first MVNO available (line 3), $i$, we compute the maximum number of aligned RBs that can be allocated on the same frequencytime slots $(n, t)$ across every BS (line 4 ), excluding the ones with no policy assigned (i.e. $S_{i, b}=0$ ). Such BSs are not considered as they will produce non-uniquely assigned RBs in those slots regardless from the RB allocation of MVNO $i$ on the remaining BSs. In the case MVNO $i$ has not RBs in any BSs (line 5), we remove it from the list of MVNOs since either its RBs have already been allocated or have never been assigned by the policy $S_{m, b}$ (line 6). For every BS, we allocate RBs to MVNO $i$ (line 9). Specifically, when the considered BS has some RBs to allocate (line 10), we sequentially allocate the computed number of aligned RBs, $s^{(i)}$, to MVNO $i$ (line 11) and we subtract them from the total amount (line 12). If the considered BS has not assigned RBs to MVNO $i$ (line 13), we choose MVNO $j$ among the ones having RBs to allocate on the considered BS such that the numerology gap from MVNO $i$ is minimized (line 14). We sequentially allocated a number of RBs to MVNO $j$ that spans a similar spectrum region as occupied by the RBs of MVNO $i$ on the other BSs (line 15). In other words, the frequency-time slots that cannot be uniquely assigned are "padded" with RBs of similar numerologies of other MVNOs in order to keep a similar RB alignment on each BS. The total number of allocated RBs to MVNO $j$ is updated accordingly (line 16). Once the allocation for MVNO $i$ is completed on every BS, if MVNO $i$ is in the last position of the MVNO list (line 19), we reverse the MVNOs order with respect to the previous iteration (line 20). This last step is performed to minimize the numerology gap between the last MVNO allocated on the current iteration and the first MVNO allocated on the next iteration. If this operation was not performed, on the next iteration, the algorithm would contiguously allocate RBs with a small numerology next to RBs with a high numerology, thus increasing the INI.

\section{Performance eValuation}

We assessed the performance of the allocation policy computed by the optimization problem (8) and the heuristic algorithm (Algorithm 1) with respect to a baseline scheme, denoted as Random policy. The latter does not account for interslice isolation and randomly allocates RBs among MVNOs. The optimal RB allocation policy was computed using the commercial solver IBM ILOG CPLEX Optimization Studio, which efficiently addresses integer non-convex quadratic problems [24]. We performed 100 independent generations of the assignment policy $S_{b, m}$ and we averaged the obtained results. Specifically, for each simulation instance, first we randomly generated $S_{m, b}$ according to $\tilde{N}_{R B}=50$ and assumed to be valid for $T=1$ time slots. Then, we computed the $\mathrm{RB}$ allocation policy using the aforementioned schemes. We averaged the results across the different iterations and we plotted the $95 \%$ confidence intervals.

We considered every numerology currently available in the 3GPP specification (see Table I) in order to better investigate INI mitigation performance in complex scenarios. Therefore, we assumed a RAN shared by up to 5 MVNOs characterized by numerologies ranging from $15 \mathrm{kHz}$ to $240 \mathrm{kHz}$. The penalty assigned to the contiguous allocation of RBs with numerology gap $k_{m, m^{\prime}}>2$ was computed with $C=5$.

\section{A. Convergence time results}

We analyze the convergence time performance of both the optimal and heuristic solution in order to assess their application in real scenarios. Fig. 3 depicts the convergence time for 3 and 5 mutually interfering BSs as the number of MVNOs with different numerologies increases from 2 to 5 . Note that we added numerologies in increasing order starting from the one with the smallest subcarrier spacing. For each case, we plot the results achieved by the optimal solution when $\alpha=0, \alpha=0.5$ 


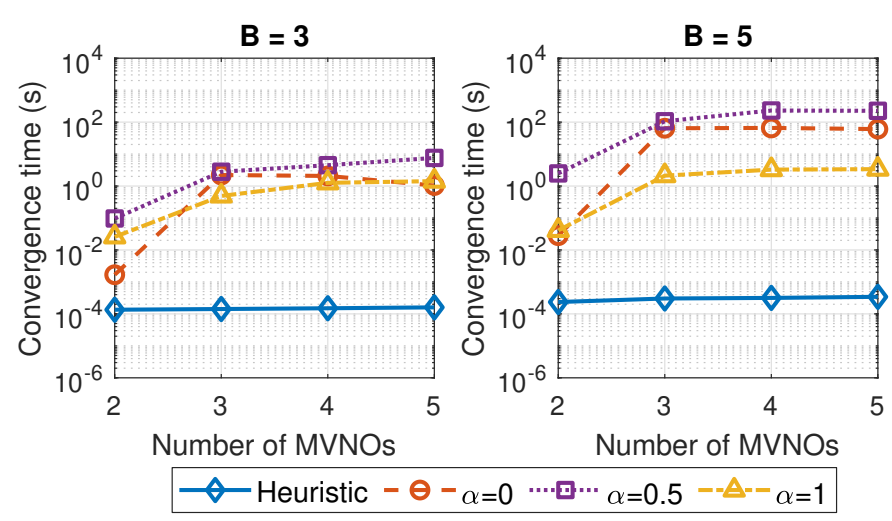

Fig. 3. Convergence time of heuristic and optimal solution for different values of BSs and MVNOs. The half-width of the confidence intervals (not shown) is smaller than $4.5 \%$ of the measured value.

and $\alpha=1$ and by the heuristic solution. We note the non-linear dependency as the number of interfering links and numerology types increases which confirms our previous analysis discussed in Section IV. Specifically, we note that $\alpha=0.5$ leads to the highest increase of the convergence time since both ICI and INI minimization terms are considered during the optimization process. Differently, setting $\alpha=0$ and $\alpha=1$ provides a more limited impact on the computational complexity as the INI and ICI term, respectively, are not simultaneously active. Moreover, we observe that, in general, the convergence time is more affected by the number of BSs due to the quadratic relationship between interfering links present in the ICI term. Regardless of the considered combination of MVNOs and BSs, the convergence time required by the proposed heuristic scheme heavily outperforms the optimal solution, thus making it suitable for practical systems.

\section{B. Inter-slice isolation results}

As we are interested in the interference power that is mutually generated among different slices, the aggregate SINR achieved by each MVNO is a meaningful metric to evaluate the inter-slice isolation. Specifically, the SINR is calculated over the RBs allocated to each MVNO using the formulation in (4). We remark that high SINR values enhance the independence between slices. This has the benefit of providing each MVNO with better radio resources that can schedule effectively among its users.

We provide the results based on the network scenario depicted in Fig. 2. Since the maximum number of interfering links is 4 independently of the number of sites, we asses the performance considering only $1 \mathrm{BS}$ that mutually interferes with 4 BSs located on 3 adjacent sites. Therefore, the RB allocation policy is computed for 5 BSs that interfere each other following the pattern described in Section III. Note that increasing the number of surrounding cells would not affect the inter-slice isolation performance as no additional interfering links are generated between the new sectors and the considered BS. For this reason, as we already discussed the scalability of the optimal solution, we can limit our analysis on a scenario that provides the highest number of interfering links with the minimum number of BSs.

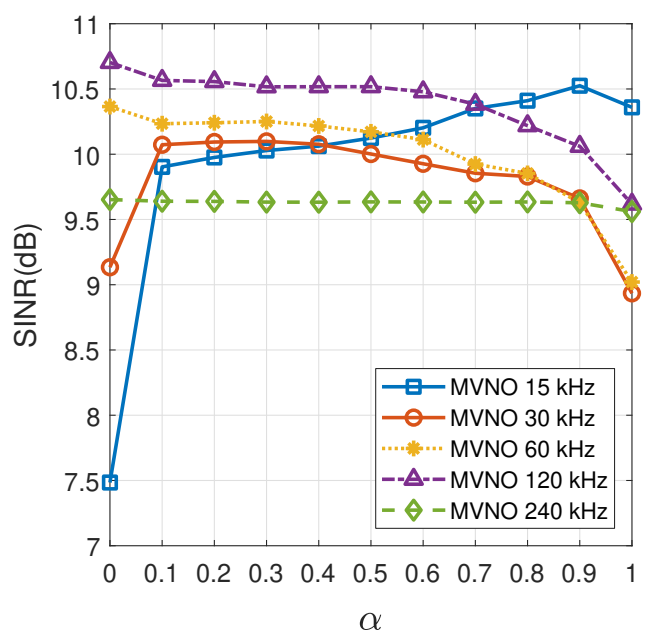

Fig. 4. SINR as a function of $\alpha$ when 5 MVNOs with numerologies of subcarrier spacing $\{15,30,60,120,240\} \mathrm{kHz}$ are active. The half-width of the confidence intervals (not shown) is smaller than $5 \%$ of the measured value.

We assume fully loaded BSs so that the available spectrum allocated to each slice is entirely exploited. Each BS spatially multiplexes 10 beams, that replicate the computed RB allocation policy. The same transmission power is used by each sector and it is equally split among the 10 beams. In the case not every beam can be spatially multiplexed due to users being located in close regions, the BS power is divided among the available beams. The power assigned to each beam is partitioned between RBs following this scheme:

- RBs with equal numerologies have equal power

- RBs of different numerologies have a power that is directly proportional to their bandwidth (for example, RB with numerology $15 \mathrm{kHz}$ has half the power of a RB with numerology $30 \mathrm{kHz}$, since it occupies half of the bandwidth).

The complete set of system parameters used in the simulations is reported in Table II, where their value was chosen following the ITU guidelines for dense-urban environments.

TABLE II

SYSTEM PARAMETERS

\begin{tabular}{lc}
\hline BS transmission power & $45 \mathrm{dBm}$ \\
Inter-site distance & $200 \mathrm{~m}$ \\
System bandwidth & $10 \mathrm{MHz}$ \\
Carrier frequency & $4 \mathrm{GHz}$ \\
Number of beams & 10 \\
Main lobe antenna gain & $10 \mathrm{~dB}$ \\
Side lobe antenna gain & $0 \mathrm{~dB}$ \\
Main lobe beamwidth & $15^{\circ}$ \\
Path loss exponent & 2.7 \\
Noise PSD & $-174 \mathrm{dBm} / \mathrm{Hz}$ \\
\hline
\end{tabular}

In Fig. 4 we show the impact of the parameter $\alpha$ on the SINR of each slice. To provide a meaningful and general analysis, we consider a scenario consisting of 5 MVNOs that employ all the numerologies defined in the NR standard and we compute the average SINR achieved by their users. We note that increasing $\alpha$ improves the performance of the 


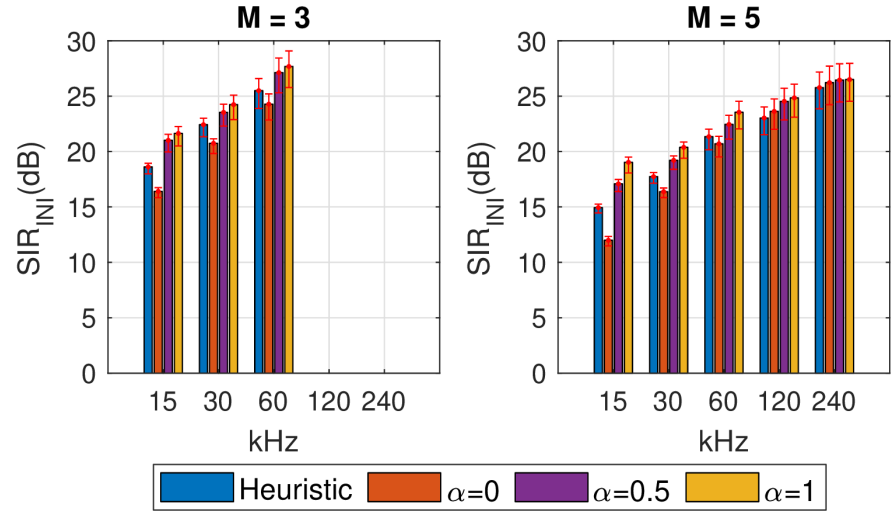

Fig. 5. Inter-numerology interference performance achieved by the heuristic solution and by the optimal solution for different $\alpha$ values when numerologies of subcarrier spacing $\{15,30,60\} \mathrm{kHz}$ and $\{15,30,60,120,240\} \mathrm{kHz}$ are active.

MVNOs with smaller numerologies ( $15 \mathrm{kHz}$ and $30 \mathrm{kHz})$. This is due to the fact that numerologies with a small subcarrier spacing are more affected by INI than higher numerologies, hence the performance improvement derived from INI minimization is more evident. Conversely, MVNOs of higher numerologies assume the highest SINR values when $\alpha$ is small as ICI dominates over INI. In general, we observe that there is no a unique $\alpha$ that allows to simultaneously maximize the SINR of every MVNO, but there are multiple values that provide different performance trade-off according to the employed numerologies. More precisely, $\alpha \in[0.1,0.5]$ ensures a balanced trade-off minimization between ICI and INI as it provides high SINR values to MVNOs with small numerologies while limiting the performance loss for MVNOs with high numerologies. Based on this analysis, we now restrict the results discussion by focusing on the RB allocation computed with $\alpha=0, \alpha=0.5$ and $\alpha=1$ that corresponds to ICI minimization, a trade-off minimization of both interference sources and INI minimization, respectively.

Due to the multi-objective nature of our problem, first we separately investigate the effect of the RB allocation policy on INI and ICI in each slice. In this analysis phase, we do not consider the noise contribution in order to better profile the performance of our approach. Analytically, we calculate the associated SIR due to INI and ICI by assuming $P_{i c i}=0$ and $P_{i n i}=0$ in (4), respectively.

Fig. 5 compares the SIR due to INI, SIR $i n i$, when 3 slices of numerology $15 \mathrm{kHz}, 30 \mathrm{kHz}$ and $60 \mathrm{kHz}(M=3)$ and when 5 slices using all the available numerologies $(M=5)$ are sharing the RAN. We observe that the optimal solution achieves higher $\operatorname{SIR}_{i n i}$ values as $\alpha$ increases, where the highest relative gain is obtained when $\alpha$ is 0.5 . This behavior is caused by the INI term in the objective function that assumes a higher priority than the ICI term when $\alpha$ gets bigger. In fact, this trend is confirmed by setting $\alpha=1$, that allows to achieve the best SIR $_{i n i}$ performance as the ICI minimization is completely neglected. Instead, the heuristic solution provides a $\mathrm{SIR}_{i n i}$ improvement that is almost comparable to $\alpha=0.5$ for every MVNO. As a general observation regarding the INI behavior related to the number of MVNOs, it is possible to note that,

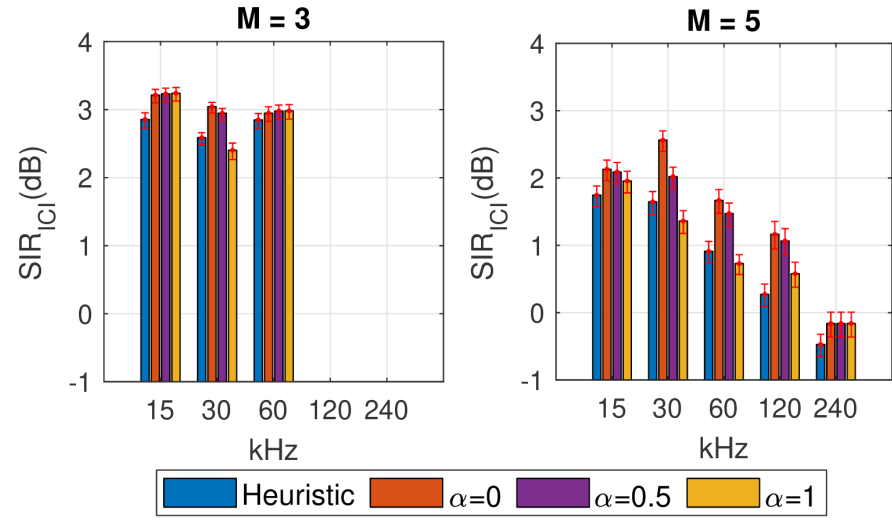

Fig. 6. Inter-cell interference performance of edge-users achieved by the heuristic solution and by the optimal solution for different $\alpha$ values when numerologies of subcarrier spacing $\{15,30,60\} \mathrm{kHz}$ and $\{15,30,60,120,240\} \mathrm{kHz}$ are active.

when the number of numerologies raises from 3 to 5 , the average $\operatorname{SIR}_{i n i}$ per slice is reduced. This fact can be easily justify considering that, as more numerologies are multiplexed over the same spectrum, the higher is the level of interference generated. Moreover, the latter asymmetrically affects each slice based on the employed numerology type. Specifically, the narrower is the subcarrier spacing, the higher is interference suffered from numerologies with a wider subcarrier spacing. For example, we note a substantial performance degradation on MVNOs with numerology $15 \mathrm{kHz}$ and $30 \mathrm{kHz}$ when the number of MVNOs is increased to 5 .

As similarly done for INI, Fig. 6 depicts the SIR due to ICI, SIR $i c i$, obtained with 3 and 5 MVNOs sharing the RAN. We plot the performance when users are located in proximity of the coverage boundaries of the serving cell in order to assess the results on a radio scenario characterized by high ICI values (in other words, when serving and interfering links have similar path loss values). In this case, we note an opposite behavior with respect to the INI case. The optimal solution provides the highest $\operatorname{SIR}_{i c i}$ when $\alpha=0$, whereas the lowest value is achieved for $\alpha=1$. This is explained by the fact that small values of $\alpha$ prioritize ICI minimization with respect to INI minimization, thus when $\alpha$ is increased the $\operatorname{SIR}_{i c i}$ is degraded. The heuristic solution provides a performance that is comparable with $\alpha=0.5$ in most cases and with $\alpha=1$ when highest order numerologies are employed (e.g. $120 \mathrm{kHz}$ and $240 \mathrm{kHz}$ ). This due to the fact that the alignment on similar time-frequency slots of such numerologies is more challenging to perform as they require wider portions of spectrum to be uniquely allocated on every BS. Generally, as similarly occurred for INI case, the average $\operatorname{SIR}_{i c i}$ per MVNOs decreases when the number of MVNOs increases. In fact, when more slices get multiplexed within the same RAN, the number of non-uniquely assigned RBs generate higher interference levels as the various MVNOs have access to a smaller spectrum.

We now analyze the performance of the optimal and heuristic solutions by accounting for both interference sources as well as the noise power and we compare it with respect 

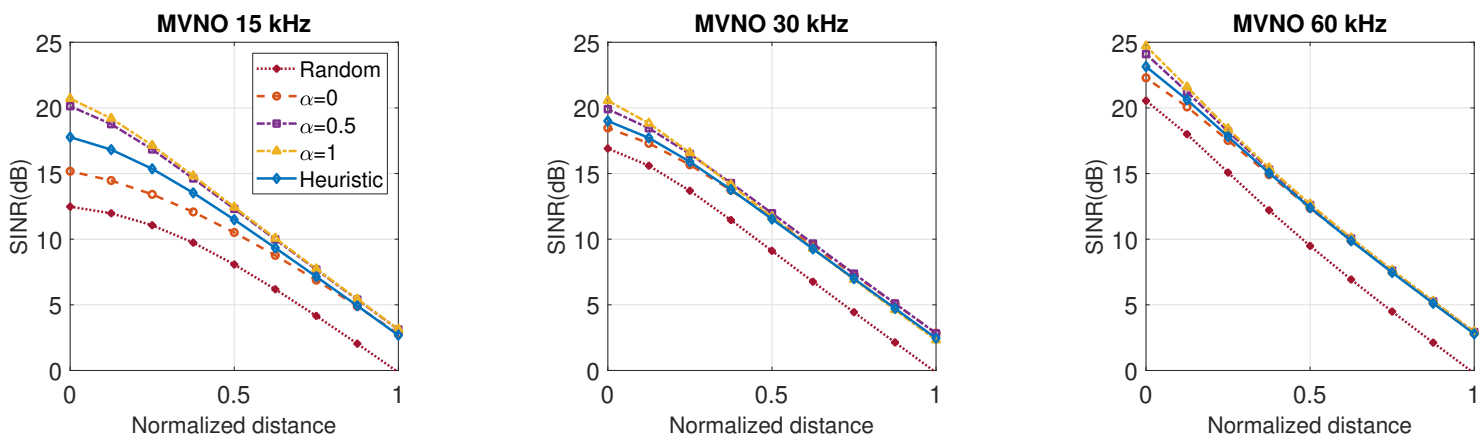

Fig. 7. SINR performance for different RB allocation policies as function of the distance from the serving BS when 3 MVNOs are considered. The half-width of the confidence intervals (not shown) is smaller than $5 \%$ of the measured value.
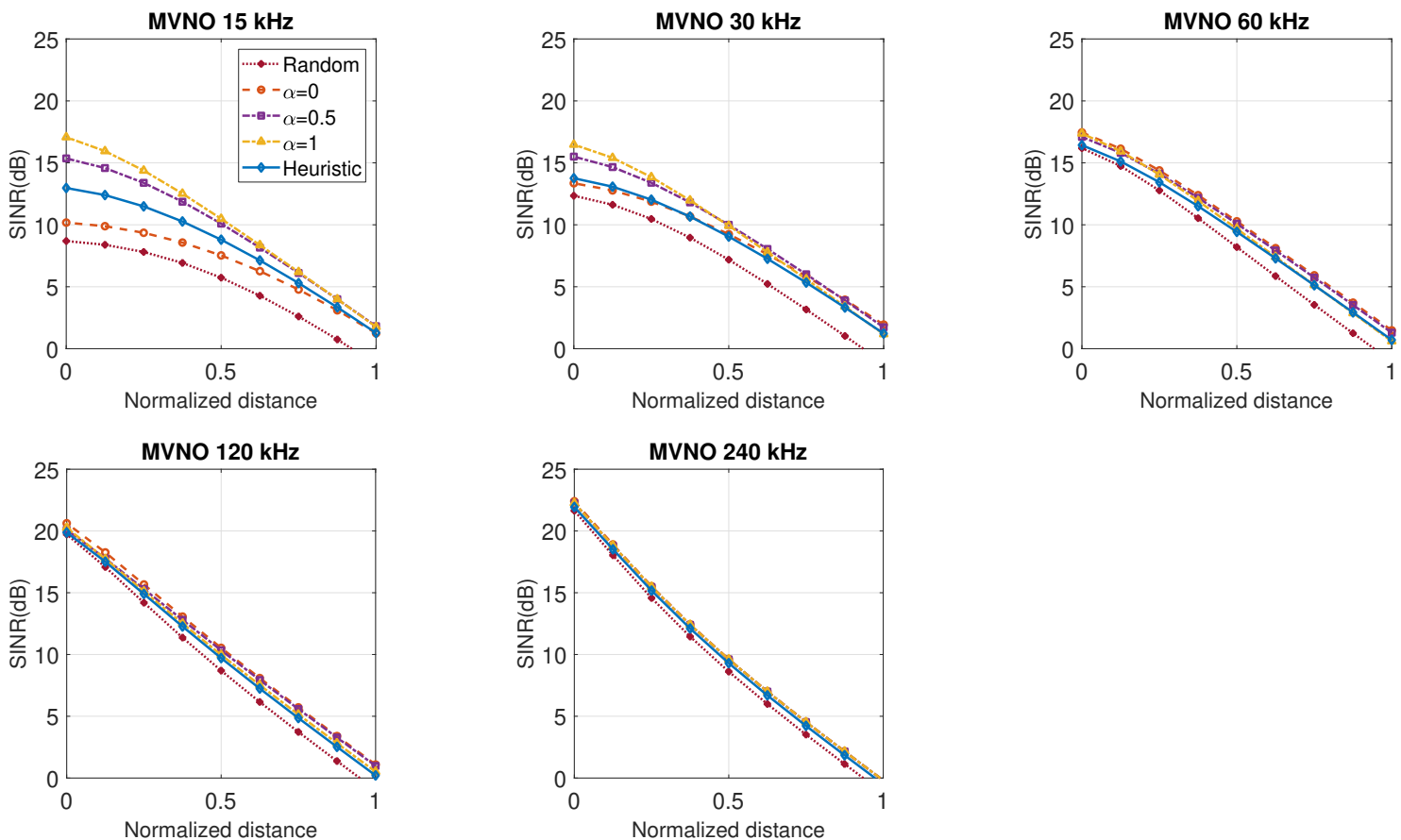

Fig. 8. SINR performance for different RB allocation policies as function of the distance from the serving BS when 5 MVNOs are considered. The half-width of the confidence intervals (not shown) is smaller than $5 \%$ of the measured value.

to the base-line policy. In detail, we evaluate the SINR associated to each MVNO as a function of the distance from the serving BS. Fig. 7 depicts the achieved SINR when 3 MVNOs are considered. We observe that the proposed approach outperforms the base-line scheme, hence it confirms the benefits in considering both INI and ICI to enforce interslice isolation. More precisely, we observe that the optimal solution provides the best SNIR performance in regions closer to the serving BS when $\alpha=1$. Differently, on the boundaries of the coverage area, the SINR improves for all MVNOs when $\alpha=0$. This trend is explained by the fact that, in the former case, INI component is dominant as the high path loss attenuation from the interfering BSs reduces the ICI, thus INI minimization ensures better results. Instead, in the latter case, ICI component is dominant as the interfering BSs are closer, thus ICI minimization is more effective. We note that $\alpha=0.5$ provides the best SINR performance in the central regions of the coverage area that are characterized by similar INI and ICI power levels. The heuristic solution achieves a performance that is comparable with the optimal solution when $\alpha \in[0,0.5]$ for all MVNOs. This behavior validates our heuristic design methodology that aims at providing a balanced minimization of both interference sources.

Finally, we plot the SINR performance when 5 MVNOs are considered in Fig. 8. The increasing number of slices leads to a higher INI level which degrades the SINR on the region closer to the serving BS. In particular, smaller numerologies $(15 \mathrm{kHz}$ and $30 \mathrm{kHz})$ benefit the most from INI minimization. Differently, highest numerologies, which are less affected by INI, have a limited SINR improvement when INI is minimized. For this reason, the optimal solution provides similar performance regardless from the employed $\alpha$ as well as a modest gain with respect to the base-line scheme for MVNOs with numerologies $120 \mathrm{kHz}$ and $240 \mathrm{kHz}$. However, this effect is further exacerbated by the limited radio bandwidth available $(10 \mathrm{MHz})$ that can hardly accommodate 
slices of such numerolgies in each BS for every time slot. Therefore, these MVNOs are more likely to be affected by inter-slice interference as they do not have assigned RBs in all interfering BSs. In any case, the base-line scheme provides very poor performance for MVNOs with smaller numerologies, hence our approach is essential to achieve the considerable SINR improvement depicted in the graph. Despite the heterogeneous behavior observed by the various MVNOs, we can observe that the heuristic solution still provides comparable performance with the optimal solution when $\alpha=0$ in each slice. We observe that a high number of numerologies hinders the heuristic solution performance as the scheme tries to align a wider set of heterogeneous RBs on similar time-frequency slots in different BSs. However, we remark that the considered scenario can be assumed as worstcase since it employs all the numerologies that are currently defined in the 3GPP NR specification. Therefore, such result is still relevant as it confirms the flexibility of the proposed heuristic scheme on different scenarios without requiring the tuning of any parameter. In conclusion, the results prove the benefit of our approach in enforcing inter-slice isolation and highlight the importance of simultaneously accounting for both ICI and INI to improve the system SINR.

\section{CONCLUSION}

We investigated a resource allocation slicing policy capable of enforcing inter-slice isolation across MVNOs. Specifically, we proposed a multi-objective optimization problem that minimized the inter-slice interference generated by interfering BSs and by the simultaneous multiplexing of RBs having different numerologies. To overcome the computation complexity of such solution, we proposed a time-efficient heuristic scheme that provides a balanced trade-off between the minimization of the two interference sources. We assessed the performance of optimal and heuristic solution under a variable number of MVNOs having different numerologies. Results revealed a consistent SINR improvement achieved by both schemes compared to a random-based RB allocation.

\section{REFERENCES}

[1] NGMN Alliance, "Description of network slicing concept," 2016. [Online]. Available: https://www.ngmn.org/publications/ description-of-network-slicing-concept.html

[2] X. Zhang, L. Zhang, P. Xiao, D. M. J. Wei, and Y. Xin, "Mixed numerologies interference analysis and inter-numerology interference cancellation for windowed OFDM systems," IEEE Transactions on Vehicular Technology, vol. 67, no. 8, pp. 7047-7061, 2018.

[3] X. Foukas, G. Patounas, A. Elmokashfi, and M. K. Marina, "Network slicing in 5G: survey and challenges," IEEE Communications Magazine, vol. 55, no. 5, pp. 94-100, 2017.

[4] S. E. Elayoubi, S. B. Jemaa, Z. Altman, and A. Galindo-Serrano, "5G RAN Slicing for Verticals: Enablers and Challenges," IEEE Communications Magazine, vol. 57, no. 1, pp. 28-34, 2019.

[5] S. Zhang, "An Overview of Network Slicing for 5G," IEEE Wireless Communications, 2019.

[6] A. Rostami, P. Ohlen, K. Wang, Z. Ghebretensae, B. Skubic, M. Santos, and A. Vidal, "Orchestration of RAN and transport networks for 5G: An SDN approach," IEEE Communications Magazine, vol. 55, no. 4, pp. 64-70, 2017.

[7] P. Caballero, A. Banchs, G. D. Veciana, X. Costa-Perez, and A. Azcorra, "Network slicing for guaranteed rate services: Admission control and resource allocation games," IEEE Transactions on Wireless Communications, vol. 17, no. 10, pp. 6419-6432, 2018.
[8] K. Zhu and E. Hossain, "Virtualization of 5G cellular networks as a hierarchical combinatorial auction," IEEE Transactions on Mobile Computing, vol. 15, no. 10, pp. 2640-2654, 2015.

[9] A. Gebremariam, M. Chowdhury, M. Usman, A. Goldsmith, and F. Granelli, "Softslice: Policy-based dynamic spectrum slicing in $5 \mathrm{~g}$ cellular networks," in 2018 IEEE International Conference on Communications (ICC). IEEE, 2018, pp. 1-6.

[10] J. J. Gang and V. Friderikos, "Optimal resource sharing in multi-tenant 5G networks," in 2018 IEEE Wireless Communications and Networking Conference (WCNC). IEEE, 2018, pp. 1-6.

[11] R. Shrivastava, K. Samdanis, and A. Bakry, "On Policy Based RAN Slicing for Emerging 5G TDD Networks," in 2018 IEEE Global Communications Conference (GLOBECOM). IEEE, 2018, pp. 1-6.

[12] B. Han, J. Lianghai, and H. D. Schotten, "Slice as an evolutionary service: Genetic optimization for inter-slice resource management in 5G networks," IEEE Access, vol. 6, pp. 33 137-33 147, 2018.

[13] C. Chang, N. Nikaein, and T. Spyropoulos, "Radio access network resource slicing for flexible service execution," in IEEE INFOCOM 2018IEEE Conference on Computer Communications Workshops (INFOCOM WKSHPS), 2018, pp. 668-673.

[14] S. D'Oro, F. Restuccia, A. Talamonti, and T. Melodia, "The slice is served: Enforcing radio access network slicing in virtualized $5 \mathrm{~g}$ systems," in IEEE INFOCOM 2019-IEEE Conference on Computer Communications, 2019, pp. 442-450.

[15] J. Shi, H. Tian, S. Fan, P. Zhao, and K. Zhao, "Hierarchical Auction and Dynamic Programming Based Resource Allocation Algorithm for 5G RAN Slicing," in 2018 24th Asia-Pacific Conference on Communications (APCC), 2018, pp. 207-212.

[16] 3GPP, "NR; Physical channels and modulation," 3rd Generation Partnership Project (3GPP), Technical Specification (TS) 38.211, 2019, version 15.5.0.

[17] ITU-R, "Guidelines for evaluation of radio interface technologies for IMT-2020," 2017. [Online]. Available: https://www.itu.int/md/ R15-SG05-C-0057

[18] T. Bai and R. W. Heath, "Coverage and rate analysis for millimeter-wave cellular networks," IEEE Transactions on Wireless Communications, vol. 14, no. 2, pp. 1100-1114, 2014.

[19] H. Elkotby and M. Vu, "Interference modeling for cellular networks under beamforming transmission," IEEE Transactions on Wireless Communications, vol. 16, no. 8, pp. 5201-5217, 2017.

[20] A. M. Hunter, J. G. Andrews, , and S. Weber, "Transmission capacity of ad hoc networks with spatial diversity," IEEE Transactions on Wireless Communications, vol. 7, no. 12, pp. 5058-5071, 2008.

[21] S. Sun, T. S. Rappaport, S. Rangan, T. A. Thomas, I. Z. K. A. Ghosh, I. Rodriguez, O. Koymen, A. Partyka, and J. Jarvelainen, "Propagation path loss models for 5G urban micro-and macro-cellular scenarios," in 2016 IEEE 83rd Vehicular Technology Conference (VTC Spring). IEEE, 2016, pp. $1-6$.

[22] A. B. Kihero, M. S. J. Solaija, A. Yazar, and H. Arslan, "InterNumerology Interference Analysis for 5G and Beyond," in 2018 IEEE Globecom Workshops (GC Wkshps). IEEE, 2018, pp. 1-6.

[23] P. M. Pardalos and S. A. Vavasis, "Quadratic programming with one negative eigenvalue is np-hard," Journal of Global Optimization, vol. 1, no. 1, pp. 15-22, 1991.

[24] IBM, "IBM ILOG CPLEX Optimization Studio CPLEX User's Manual," 2016. [Online]. Available: https://www.ibm.com/support/knowledgecenter/SSSA5P_12.7.0/ ilog.odms.studio.help/pdf/usrcplex.pdf 